\title{
An Iteration-Based Simulation Method for Getting Semi- Symbolic Solution of Non-coherent FSK/ASK System by Using Computer Algebra Systems
}

\author{
Vladimir MLADENOVIC ${ }^{1 *}$, Sergey MAKOV ${ }^{2}$, Viacheslav VORONIN ${ }^{2}$, Miroslav LUTOVAC \\ ${ }^{1}$ Faculty of Technical Sciences in Cacak, University of Kragujevac, \\ Svetog Save 65, Cacak, 32000, Serbia, \\ vladimir.mladenovic@ftn.kg.ac.rs (*corresponding author) \\ ${ }^{2}$ Don State Technical University, \\ Shevchenko 147, Shakhty 346500, Rostov region, Russia, \\ makovserg@yandex.ru, voroninslava@gmail.com \\ ${ }^{3}$ Singidunum University, \\ Belgrade, Danijelova 32, Belgrade, 11000, Serbia, \\ lutovac@gmail.com.
}

\begin{abstract}
The paper presents a development of an IBSM (Iteration-Based Simulation Method) to obtain semi-symbolic solutions of digital telecommunication non-coherent FSK/ASK system by using computer algebra systems. These solutions are applied for derivation of the symbol error probability (SEP) needed for quantitative and qualitative analysis of performances of telecommunication systems in optimization and design of low-complexity implementation into highcomplexity structures. Various software tools, used in telecommunications for calculating, designing and analysing, have been developed from the viewpoint of numeric-only algorithms. But, many shortcomings cannot be neglected, such as generating of a great amount of numeric data, losing insight into the phenomenon of investigation, and numerical computation manipulates with numerical values. For these reasons, IBSM provides design, optimization, manipulation, and simplification by introducing a new parameter of iteration. The IBSM is applied to aforementioned system when the uncorrelated and correlated noise and the interferences are present in both upper and lower frequencies, respectively. The impact of number of iterations, number of bits, correlation coefficient and different values of interferences to symbol error probability are derived on semi-symbolic forms. Making use of IBSM gives an opportunity to solve very complex analyses and derive many sophisticated conclusions.
\end{abstract}

Keywords: Closed-form solution, Wolfram language, Rayleigh fading, Software tools, Computer algebra system

\section{Introduction}

The rapid growth of wireless communication can be seen through the increasing number of users and the exploitation of the frequency band in which the information is exchanged. Fixed-to-fixed channels, fixed-to-vehicle channels, vehicle-to-vehicle channels, mobileto-mobile communications, moving scatterers, vehicular communications are only some of challenges that have become the up to date subject of research in the growing mobile industry [1]. All aforementioned communication models are analysed using statistical methods. One of the phenomena is the Rayleigh fading that occurs in urban environments. It represents the propagation of electromagnetic wave when there is no a dominant component in the propagation, and received signal is sum of reflected signals. Typically, this fading is presented in places with buildings and high objects. Almost all analyses are performed using software tools that are based on the numeric-only algorithms. Using them, many graphic characteristics can be illustrated, but the behaviour of systems and processes, that describe the performance, are impossible to observe. On the other hand, the fact is ignored that numerical computation generates a large amount of data, which may sometimes lead to erroneous results [14]. They may be the result of the finite word length in the records, or errors during shortenings of numbers in fractions for example. These ways do not provide possibility to manipulate with analytical expressions.

To overcome these problems, the use of symbolic processing, which formulates a new method called the iteration-based simulation method, is introduced [10]. Symbolic processing provides advanced solutions to all the existing gaps and pushes the boundaries to new models of survey and design. The primary aspiration is speeding up in design and analysis of lowcomplexity implementation into highcomplexity structures, which are provided by significantly increasing of processor's power and higher storage space, so the expert-based knowledge is necessary in areas such as theory 
of systems, signal processing, telecommunications, and software engineering [14]. Methods of symbolic processing are known in the industry in academic fields. They are used as an aid in the design of electronic circuits and systems in the industry. In academic institutions, they are used as auxiliary tools in the classroom. Mathematical operations with errors-free concept can be performed to write simulation code for symbolic processing, even to find errors in the manual execution of published results [9]. Also, symbolic processing can be used to find expression in closed-form, and when a large number of iterations exists, or functions errors occurred. The efficiency of symbolic processing becomes more important if the systems and signals are more complex [6].

This paper is structured as follows. In section 2, the state-of-art is presented from viewpoint of kind of simulation and subject of researches. In section 3, the approach of knowledge programming using computer algebra system and Wolfram language is introduced. Section 4 describes the reference model and analysis of communication system. In section 5 , the iteration-based simulation method is explained and Wolfram language is used to model it. Code-deriving closed-form solutions of SEP are presented in section 6 by using iterationbased simulation method. Finally, section 7 provides symbolic-based optimization procedure and convergence testing to get closed-form solutions, and transformation to semi-symbolic forms to obtain final results.

\section{Related Work}

Typically, leading role in simulations of many processes and systems has numerical tools. However, the manipulation with expressions by hand is necessary before each simulation. Often, such an approach can be time consuming, and the obtained numerical results cannot be satisfactorily. These simulation methods are used in many cases. For example, the frequency-nonselective mobile Rayleigh fading channels are obtained for modelling and simulating in paper [3] with closed-form expressions of the classes of sum-of-cissoids processes. Often, it can be obtained when solution exists through special function, but it is known that the closed-form expression is in the background [4]. Many of approaches are applied to develop mobile channel models by a finite sum of multipath propagation components [13]. A several approaches have been proposed in [5] for these studies to reduce the drawbacks of this algorithm. The application of algebraic computer system is presented in a number of papers [12] explaining how automated symbolic solving problem in different areas can be used to solve without manual execution and verification of results. Of course, there are limitations and it should be always bear in mind that the software programs that use symbolic tools can provide solutions only if knowledge is entered into the used software tools, inasmuch real solutions exist [6], [8].

\section{Computer Algebra System and Wolfram Language}

There are two steps in the engineering sciences to formulate solving of problems. The first step is to understand various engineering fields such as processes and systems. Secondly, the knowledge transfer into the corresponding software, which commonly uses procedures for solving. The best known language to encapsulate expert knowledge in software is Wolfram language [15].

Let us define the key definitions that are the initial formulation of the mathematical descriptions of the process and systems. An equation is said to be a closed-form solution if it solves a given problem in terms of functions and mathematical operations from a given generally-accepted set [2]. On the other side, the closed-form expression represents an implicit solution that is contained in a mathematical expression. In other words, closed-form expression provides implicitly or insufficient solution of a process and systems while closed-form solution shows explicitly solution. Computer algebra system is software that provides environment and support for all manipulations, processing, solving, and many derivations of mathematical expressions. Symbolic processing concerns the development, implementation and application of algorithms that manipulate and analyse mathematical expressions. The Wolfram language provides many built-in functions that are used for term rewriting. Building, programming and execution of many processes are comprehended as everything-expressed structures. It is very easy to check if automated 
derivation is generating the same result with simplification rules and by rearranging expressions [11]. Calculation of the expressions is often very complex and arduous, and sometimes there is no final result. The main idea is to focus on examples for users who use software for analysis or an exemplary document, processing or simulation.

\section{The Reference Model and Analysis}

Before derivation of IBSM, let us describe a system that will be analyzed and where the method will be applied.

Non-coherent FSK/ASK (Frequency-Shift Keying/Amplitude-Shift Keying) is a modulation scheme used to send digital information between digital equipment. The data is transmitted by non-coherent system without carrier in a binary manner to one or the other of two discrete frequencies. One frequency is designated as "upper" frequency and the other as the "lower" frequency. The "upper" and "lower" correspond to symbols of binary zero-zero, zero-one, one-zero, one-one, respectively. By convention, upper corresponds to the higher radio frequency. The specifics of function for propagation environment are important to determine such as PDF (Probability Density Function) of the receiving signal and the CDF (Cumulative Probability Function). The parameters of the system are described analytically in closed-form solutions based on closed-form expressions of SEP.

This approach provides a better insight into manipulations of all parameters and the progressing of processes that are necessary to properly transmit information unlike traditional numerical methods. The Wolfram language is used for this approach that applies the symbolic tools. In this way, the detection parameters are obtained in the final analytical form which often is not the case. The characteristics of the system are shown using numerical data on closed-form solutions that present the special case of symbolic calculations.

Entire system is analyzed with the interference, denoted as $i_{1}(\mathrm{t})=A_{0} \cdot \cos \left(\omega_{0} \mathrm{t}\right)+A_{1} \cdot \cos \left(\omega_{1} \mathrm{t}\right)$. The noise is narrowband denoted as $n_{\mathrm{k}}(\mathrm{t})=$ $x_{\mathrm{k}}(\mathrm{t}) \cdot \cos \left(\omega_{\mathrm{k}} \mathrm{t}\right)-y_{\mathrm{k}} \cdot \sin \left(\omega_{\mathrm{k}} \mathrm{t}\right)$. Figure 1 presents looks of non-coherent FSK/ASK.

The general statement of observed system has been explained in detail in [11]. Non-coherent detection is present on both branches of the receiver. The upper branch contains matched filter with frequency $\omega_{0}$, and lower branch contains matched filter with frequency $\omega_{1}$. Each branch is composed by an envelope phase, and further signal passes to subtraction circuit, and difference of signals goes to the sampler. Information is sent by two symbols (named codewords) on frequencies $\omega_{0}$ and $\omega_{1}$ with equal probability. The interference with the amplitude $A_{1}$ on the frequency of $\omega_{0}$ has been present in the upper branch, while it does not exist on the lower branch at frequency $\omega_{1}$. In one case the noise is correlated, and the coefficient of correlation is marked by $R$.

In second case the noise is uncorrelated $(R=0)$. Transmitter sends codewords where each one corresponds to hypothesis. For example, codeword 00 corresponds to hypothesis $H_{0} H_{0}$, codeword 01 corresponds to hypothesis $H_{0} H_{1}$, etc.

General form of hypothesis on the upper branch is:

$$
\begin{aligned}
& H_{k}: \\
& \begin{aligned}
w_{k} & =(-1)^{k+1} A \cos \omega_{0} t+A_{0} \cos \left(\omega_{0} t+\theta\right)+ \\
& +x_{k} \cos \omega_{0} t-y_{k} \sin \omega_{0} t= \\
& =z_{U p k} \cos \left(\omega_{0} t+\phi_{U p k}\right)
\end{aligned}
\end{aligned}
$$

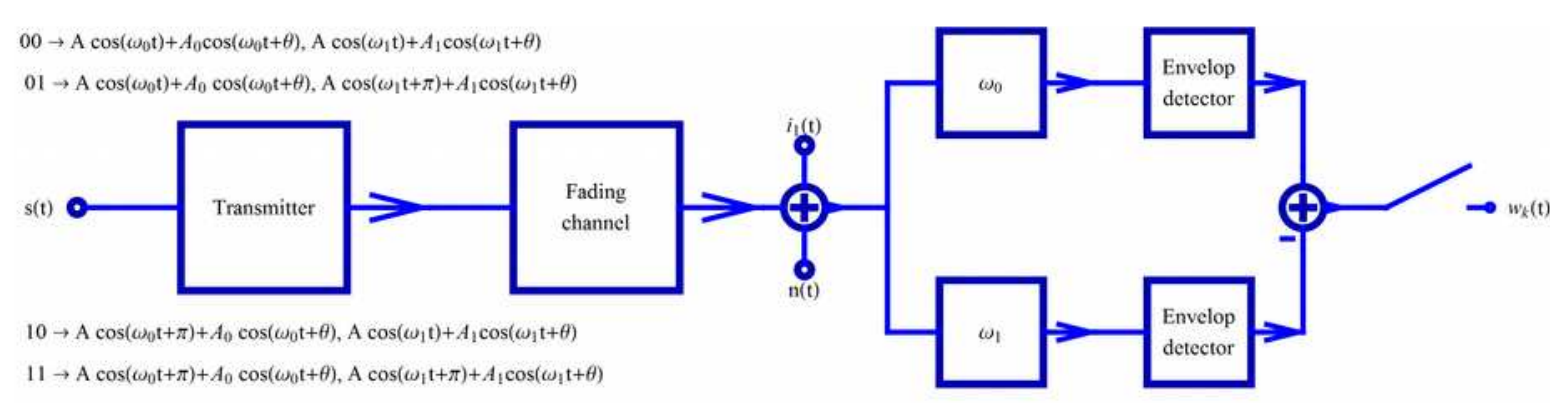

Figure 1. Non-coherent FSK/ASK system with narrow band noise and interference $i_{1}(\mathrm{t})$ 
In equation (1) $k=0,1$ and index " $U p$ " denotes the upper branch. Similarly, general form of hypothesis on the lower branch is:

$$
\begin{aligned}
& H_{k}: \\
& \begin{aligned}
w_{k} & =(-1)^{k} A \cos \omega_{1} t+A_{1} \cos \left(\omega_{1} t+\theta\right)+ \\
& +x_{k} \cos \omega_{1} t-y_{k} \sin \omega_{1} t= \\
& =z_{D w k} \cos \left(\omega_{1} t+\phi_{D w k}\right)
\end{aligned}
\end{aligned}
$$

In equation (2) $k=0,1$ and index " $L w$ " denotes the lower branch.

By substituting

$$
\begin{aligned}
& (-1)^{k+1} A+A_{0} \cos \theta+x_{k}=z_{U p k} \cos \phi_{U p k} \\
& y_{k}=-z_{U p k} \sin \phi_{U p k}
\end{aligned}
$$

The $z_{\mathrm{Upk}}$ and $\varphi_{\mathrm{Upk}}(k=0,1)$ are envelops and phases of the input signal for the upper branch follows

$$
\begin{aligned}
& z_{U p k}=\sqrt{\left((-1)^{k+1} A+A_{0}+x_{k}\right)^{2}+y_{k^{2}}} \\
& \phi_{U p k}=-\arctan \frac{y_{k}}{(-1)^{k+1} A+A_{0}+x_{k}}
\end{aligned}
$$

General form of the conditional joint probability density function results from jointly Gaussian density function for $n$ random variables. Also, it is known as a general multivariate normal density for $d$ dimensions given by

$$
\begin{aligned}
f(x ; \mu, \Sigma) & =\frac{1}{(2 \pi)^{d / 2}|\Sigma|^{1 / 2}} \times \\
& \times \exp \left\{-\frac{1}{2}(x-\mu)^{T} \cdot \Sigma^{-1} \cdot(x-\mu)\right\}
\end{aligned}
$$

where $x$ is a $d$-component column vector, $\mu$ is the column vector of values, and $\Sigma$ is the $d \times d$ covariance matrix. The superscript $T$ represents the transpose of an array, and the notation $|\cdot|$ denotes the determinant of a matrix [7].

So, conditional joint probability density function for two random variables is:

$$
\begin{array}{r}
p\left(x_{0}, x_{1}, y_{0}, y_{1}\right)=\frac{1}{4 \pi^{2} \sigma^{4} \sqrt{1-R^{2}}} \times \\
\times e^{-\frac{x_{0}^{2}+x_{1}^{2}+y_{0}^{2}+y_{1}^{2}-2 R\left(x_{0} x_{1}+y_{0} y_{1}\right)}{2 \sigma^{2}\left(1-R^{2}\right)}}
\end{array}
$$

The $R$ is coefficient of correlation and $\sigma$ is variance.

For solving of general settings, Jacobian for upper and lower branch is needed to find. Jacobian for upper branch is

$$
\begin{aligned}
& |J|_{U p}=\frac{\partial\left(x_{0}, y_{0}, x_{1}, y_{1}\right)}{\partial\left(z_{U p 1}, \phi_{U p 1}, z_{U p 2}, \phi_{U p 2}\right)}= \\
& \frac{\partial x_{0}}{\partial z_{U p 1}} \quad \frac{\partial y_{0}}{\partial z_{U p 1}} \quad \frac{\partial x_{1}}{\partial z_{U p}} \quad \frac{\partial y_{1}}{\partial z_{U p 1}} \\
& =\mid \begin{array}{llll}
\frac{\partial x_{0}}{\partial \phi_{U p 1}} & \frac{\partial y_{0}}{\partial \phi_{U p 1}} & \frac{\partial x_{1}}{\partial \phi_{U p} 1} & \frac{\partial y_{1}}{\partial \phi_{U p 1}} \\
\frac{\partial x_{0}}{\partial y_{0}} & \frac{\partial x_{1}}{\partial y_{1}}
\end{array} \\
& \frac{\partial x_{0}}{\partial z_{U p 2}} \frac{\partial y_{0}}{\partial z_{U p 2}} \quad \frac{\partial x_{1}}{\partial z_{U p 2}} \quad \frac{\partial y_{1}}{\partial z_{U p 2}} \\
& \frac{\partial x_{0}}{\partial \phi_{U p 2}} \frac{\partial y_{0}}{\partial \phi_{U p 2}} \frac{\partial x_{1}}{\partial \phi_{U p 2}} \frac{\partial y_{1}}{\partial \phi_{U p 2}} \\
& =z_{U p 1} z_{U p 2}
\end{aligned}
$$

Respectively, the Jacobian for lower branch is $|J|_{D w}=z_{L w 1} \cdot z_{L w 2}$.

The next step is finding of condition joint probability density function (JPDF). For upper and lower branch, respectively, the condition joint probability density function is

$$
\begin{aligned}
& p_{m n}\left(z_{U p 1}, z_{U p 2}, \phi_{U p 1}, \phi_{U p 2} / A\right)= \\
& \quad=|J|_{U p} \cdot p_{m n}\left(x_{0}, x_{1}, y_{0}, y_{1}\right) \\
& p_{m n}\left(z_{D w 1}, z_{D w 2}, \phi_{D w 1}, \phi_{D w 2} / A\right)= \\
& \quad=|J|_{L w} \cdot p_{m n}\left(x_{0}, x_{1}, y_{0}, y_{1}\right)
\end{aligned}
$$

here $(m, n)=(0,1)$. The joint probability density function (JPDF) is obtained by integrating condition joint probability density function for all amplitudes of $A$. The following equitation applies to the upper branch:

$$
\begin{aligned}
& p_{m n}\left(z_{U p 1}, z_{U p 2}, \phi_{U p 1}, \phi_{U p 2}\right)= \\
& =\int_{0}^{\infty} p_{m n}\left(z_{U p 1}, z_{U p 2}, \phi_{U p 1}, \phi_{U p 2} / A\right) p(A) d A
\end{aligned}
$$

and to the lower branch is:

$$
\begin{aligned}
& p_{m n}\left(z_{D w 1}, z_{D w 2}, \phi_{D w 1}, \phi_{D w 2}\right)= \\
& =\int_{0}^{\infty} p_{m n}\left(z_{D w 1}, z_{D w 2}, \phi_{D w 1}, \phi_{D w 2} / A\right) p(A) d A
\end{aligned}
$$

here $(m, n)=(0,1)$.

Now, it is necessary to obtain function of phase distribution. It is calculated by integrating JPDF for all envelops $z_{U p 1}, z_{U p 2}$ for the upper branch, and $z_{L w 1}, z_{L w 2}$ for the lower branch, respectively. Upper branch is:

$$
\begin{aligned}
& p_{m n}\left(\phi_{U p 1}, \phi_{U p 2}\right)= \\
& =\int_{0}^{\infty} \int_{0}^{\infty} d z_{U p 1} d z_{U p 2} p_{m n}\left(z_{U p 1}, z_{U p 2}, \phi_{U p 1}, \phi_{U p 2}\right)
\end{aligned}
$$

Lower branch is:

$$
\begin{aligned}
& p_{m n}\left(\phi_{L w 1}, \phi_{L w 2}\right)= \\
& =\int_{0}^{\infty} \int_{0}^{\infty} d z_{L w 1} d z_{L w 2} p_{m n}\left(z_{L w}, z_{L w 2}, \phi_{L w 1}, \phi L w 2\right)
\end{aligned}
$$


Sixteen events occur on the input of the receiver [11]. When hypothesis $H_{0} H_{0}$ is present at the output of the transmitter, codeword 00 is detected at the input of the receiver and the event $D_{0} D_{0}$ is applied, when hypothesis $H_{0} H_{1}$ is present at the output of the transmitter, codeword 01 is detected at the input of the receiver and the event $D_{0} D_{1}$ is applied, etc. At the input of the receiver, the following is detected:

$$
\begin{aligned}
& p\left(D_{m} D_{n} / H_{i} H_{j}\right)= \\
& =\int_{\frac{(-1)^{m+1}}{2} \pi}^{\frac{(m+1)}{2} \pi} d \phi_{U p 1} \int_{\frac{(-1)^{n+1}}{2} \pi}^{\frac{(n+1)}{2} \pi} d \phi_{U p 2} \int_{\frac{(-1)^{m+1}}{2} \pi}^{\frac{(m+1)}{2} \pi} d \phi_{L w 1} \times \\
& \frac{(n+1)}{2} \pi \\
& \times \int_{\frac{(-1)^{n+1}}{2} \pi}^{2} p_{i j}\left(\phi_{U p 1}, \phi_{U p 2}\right) p_{i j}\left(\phi_{L w 1}, \phi_{L w 2}\right) d \phi_{L w 2}
\end{aligned}
$$

where $(m, n, i, j)=(0,1)$. The total SEP is:

$$
\begin{aligned}
& P_{e}=1-\sum_{i=0}^{1} \sum_{j=0}^{1} P\left(H_{i} H_{j} D_{i} D_{j}\right) \\
& P\left(H_{i} H_{j} D_{i} D_{j}\right)= \\
& =p\left(D_{i} D_{j} / H_{i} H_{j}\right) \cdot P\left(H_{i} H_{j}\right) \\
& P\left(H_{i} H_{j}\right)=P\left(H_{i}\right) P\left(H_{j}\right)=\frac{1}{2} \cdot \frac{1}{2}=\frac{1}{4}
\end{aligned}
$$

here $(i, j)=(0,1)$. Such approach shows a symbolic representation of resolving the general way for every design, modelling and simulation, and numerical approach is only a special case of symbolic calculation.

\section{The Iteration-based Simulation Method and Wolfram Language Modelling}

The iteration-based simulation method represents a simplification of complex algebraic expressions that is acceptable for analysis and reduced to a closed-form solution. So, there are integrals in the most analyses, we approach them by elementary calculating when the integrals are presented using Riemann sum. We develop almost complete algorithm trying to make low-complexity implementation into high-complexity structure. Riemann sum in Wolfram language defined as follows:

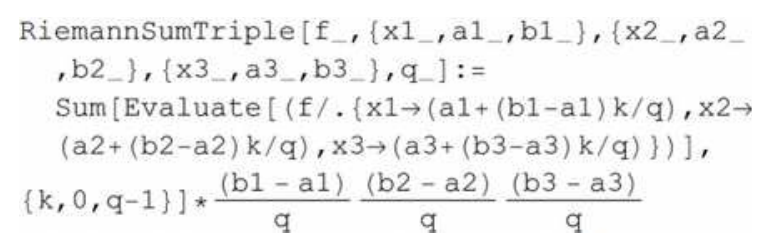

Figure 2. Riemann sum defined using Wolfram language

Definition of triple integral over Riemann sum is done by assigning the function name RiemannSumTriple. Form in Figure 2 shows triple integral where $f$ is marked as function, $\times 1, x 2$, and $x 3$ are variables, a1, a2 and $a 3$, are the below, and b1, b2 and b3 are the upper limits of integral, and $\mathrm{q}$ is iteration. In this way, defined integral is used to obtain closed-form solutions performed in complex mathematical formulas. Assigning one variable or more variables to function is done with $:=$ means with delayed values. The assigned variables are in an unevaluated form until the function is called that allocates concrete variables or variable values. Every time, when the function appears, it is replaced by variables, evaluated afresh each time.

\section{Code-deriving Closed-form Solutions of Symbol Error Probability by using an Iteration- based Simulation Method}

In this section we encapsulate aforementioned procedure. The cases are observed when the noise is correlated and uncorrelated. Coded formulation is developed by using Wolfram language. So, the Wolfram language code, in the next figure, represents expanded expression (6) as closed-form expression:

$$
\begin{aligned}
& \mathrm{p}\left[\mathrm{k}_{-}, 1_{-}, \mathrm{z} 0_{-}, \mathrm{z} 1_{-}, \mathrm{s}_{-}, \mathrm{R}_{-}, \mathrm{A}_{-}, \mathrm{f0} 0_{-}, \mathrm{f} 1_{-}, \mathrm{A} 0_{-}, \mathrm{A} 1_{-}, \text {teta }\right]:=\frac{\mathrm{z} 0 * \mathrm{z} 1}{4 * \pi^{2} *(\mathrm{~s})^{4} * \sqrt{1-\mathrm{R}^{2}} * \operatorname{Exp}[} \\
& \frac{1}{2(\mathrm{~s})^{2}\left(1-\mathrm{R}^{2}\right)}\left(\left(\mathrm{z} 0 * \operatorname{Cos}[\mathrm{f} 0]-(-1)^{\mathrm{k}+1} * \mathrm{~A}-\mathrm{A} 0 * \operatorname{Cos}[\text { teta }]\right)^{2}+\left(\mathrm{z} 1 * \operatorname{Cos}[\mathrm{f} 1]-(-1)^{1} \mathrm{~A}\right.\right. \\
& -\mathrm{A} 1 * \operatorname{Cos}[\text { teta }])^{2}+(\mathrm{z} 0 * \operatorname{Sin}[\mathrm{f} 0]+\mathrm{A} 0 * \operatorname{Sin}[\text { teta }])^{2}+(-\mathrm{z} 1 * \operatorname{Sin}[\mathrm{f} 1]+\mathrm{A} 1 * \operatorname{Sin}[\operatorname{teta}])^{2}-2 * \mathrm{R} * \\
& \left(( \mathrm { z } 0 * \operatorname { C o s } [ \mathrm { f } 0 ] - ( - 1 ) ^ { \mathrm { k } + 1 } * \mathrm { A } - \mathrm { A } 0 * \operatorname { C o s } [ \text { teta } ] ) * \left(\mathrm{z} 1 * \operatorname{Cos}[\mathrm{f} 1]-(-1)^{1} \mathrm{~A}\right.\right. \\
& -\mathrm{A} 1 * \operatorname{Cos}[\text { teta }])+\left(( \mathrm { z } 0 * \operatorname { S i n } [ \mathrm { f } 0 ] + \mathrm { A } 0 * \operatorname { S i n } [ \text { teta } ] ) * \left(\mathrm{z} 1 * \operatorname{Cos}[\mathrm{f} 1]-(-1)^{1} \mathrm{~A}\right.\right. \\
& -\mathrm{A} 1 * \operatorname{Cos}[\text { teta }]))))]
\end{aligned}
$$

Figure 3. Condition joint probability density function using Wolfram language 
Interference $i_{1}(\mathrm{t})$ is described with amplitude $A_{0}$ and $A_{1}$, and phase $\theta$, and it is present on frequencies $\omega_{0}$ and $\omega_{1}$, i.e. upper and below branches, respectively. We assume that the phase is uniform distributed. During coding we put amplitude $A_{1}$ to all closed-form expressions to examine how much it impacts to real detection and final SEP from viewpoint of iteration parameter. The amplitude $A$ carries useful information and behaviours by the Rayleigh distribution. Mentioned processes are described in Figure 4 typewritten on Wolfram language as follows:

$$
\begin{aligned}
\text { pTeta }: & =\frac{1}{2 \pi} \\
\text { pARayleght }\left[A_{-}, s_{-}\right]: & =\frac{A}{\left(s^{2}\right)^{2}} * \operatorname{Exp}\left[-\frac{A^{2}}{2(s)^{2}}\right]
\end{aligned}
$$

Figure 4. Statistical distribution for phase and Rayleigh distribution

The term Teta denotes phase $\theta$, and $s$ is variance $\sigma$.

In the next step we define probability density function according to expressions (7) to (13).

Displayed code involves $R$ as coefficient of correlation, $q$ as iteration, $s$ as variance, $z 0$ and $\mathrm{z} 1$ as envelops, $\mathrm{fO}$ and $\mathrm{fl}$ as phases. This intermediate step is used to define the overall probability of error. Wolfram code declares probability density function and provides the skeleton form of probability density function expression. These sequences are used in all further processing. The probability density function is defined by PDensityFunRayleght. It is important to note that the iteration parameter $q$ exists in the probability density function expression. This code is written on Figure 5. The theoretically defined formulations from (15) to (17) are used for getting off the symbol error probability. The final closed-form expression cannot be obtained by performing by hand because of the complexity of mathematical forms, and their simulation can be done by numerical-based software. These calculations are programstructured loop, and it is often difficult to predict the total number of iterations in the loop. It should be noted that more complex analysis in this environment is often a long time consuming, so, it is not possible to perform the simulation. Computer algebra system delivers more capabilities, and it is used for deeper understanding of observed process, and preparing it to simulate and design.

\section{Symbolic-based Optimization Procedure, Convergence Testing and Results}

The main task of IBSM is to provide a closedform solution of probability of error (in our case symbolic error probability) using the least number of iterations, which the classical numerical-only algorithms do not provide. The obtained expression can be exploited for further manipulation, solving other phenomena as well as to provide detailed insight into the observed process. In this way, we get very clear impact of certain parameters on the probability error, and the way of making the corresponding optimization. Obtaining an optimal solutions is performed by converge examination of the IBSM. Convergence can be monitored with precision by specifying parameter of iterations, and it leads to influence the final results: accuracy, execution time of method, efficiencybased reasoning, simplification of functions, and give general formulas as answers, and various other manipulations.

Using previous defined probability density function with Wolfram code on Figure 5, we perform knowledge implementation to get SEP. For this purpose, we encapsulate PDF in function PeRayleght.

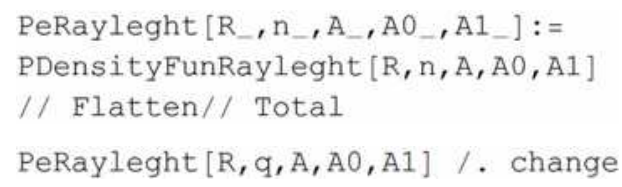

Figure 6. Defining of expression of total symbol error probability in closed-form

$$
\begin{aligned}
& \text { PDensityFunRayleght }[R, q, A, A 0, A 1][[1]] / \text {. change // Simplify } \\
& \frac{1}{32 \pi q^{4} \sqrt{1-R^{2}} \sigma^{6}} A E^{\wedge}\left(-\frac{1}{2 q^{2}\left(-1+R^{2}\right) \sigma^{2}}\left(2+A^{2} q^{2}-R+2 A^{2} q^{2} R+A^{2} q^{2} R^{2}+2 A q R \operatorname{Cos}\left[\frac{\pi}{q}\right]+R \operatorname{Cos}\left[\frac{2 \pi}{q}\right]+R\right.\right. \\
& \operatorname{Sin}\left[\frac{2 \pi}{q}\right]+q^{2} A_{0}^{2}-q\left(2-R+R \operatorname{Cos}\left[\frac{2 \pi}{q}\right]+2 A q(1+R) \operatorname{Sin}\left[\frac{\pi}{q}\right]+R \operatorname{Sin}\left[\frac{2 \pi}{q}\right]\right) A_{1}+q^{2} A_{1}^{2}+q A_{0}\left(R+2 A q R \operatorname{Cos}\left[\frac{\pi}{q}\right]+2\right. \\
& \left.\left.\left.\operatorname{Cos}\left[\frac{2 \pi}{q}\right]-R \operatorname{Cos}\left[\frac{2 \pi}{q}\right]+2 A q \operatorname{Sin}\left[\frac{\pi}{q}\right]+2 A q R \operatorname{Sin}\left[\frac{\pi}{q}\right]+R \operatorname{Sin}\left[\frac{2 \pi}{q}\right]+q R\left(-1+\operatorname{Cos}\left[\frac{2 \pi}{q}\right]-\operatorname{Sin}\left[\frac{2 \pi}{q}\right]\right) A_{1}\right)\right)\right)
\end{aligned}
$$

Figure 5. Wolfram code for defining probability density function 
Also, it should be noted that there is an iteration parameter in this expression, so it allows that this expression is in the closedform. The instruction change presents set of unique symbols used in defined distribution as:

change $=\left\{z 0->z_{0}, z 1->z_{1}, s->\sigma, f 0->\psi_{0}\right.$,

f1-> $\psi_{1}, \mathrm{~A} 0->\mathrm{A}_{0}, \mathrm{~A} 1->\mathrm{A}_{1}$, teta $\left.\left.->\theta\right]\right\}$;

The full expression of closed-form expression symbol error probability in closed-form expression is shown on Figure 7.

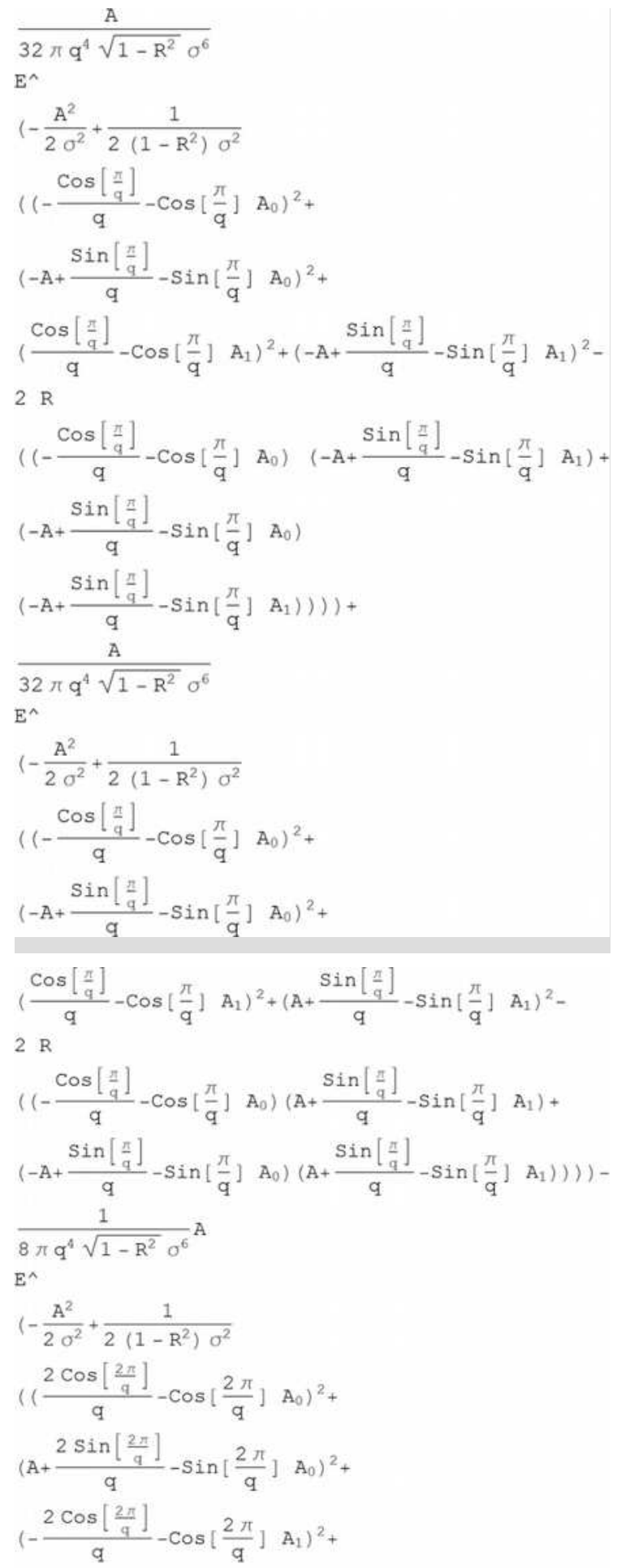

$\left(-A+\frac{2 \sin \left[\frac{2 \pi}{q}\right]}{q}-\sin \left[\frac{2 \pi}{q}\right] A_{1}\right)^{2}-$

$2 \mathrm{R}$

$\left(\left(\frac{2 \operatorname{Cos}\left[\frac{2 \pi}{q}\right]}{q}-\operatorname{Cos}\left[\frac{2 \pi}{q}\right] A_{0}\right)\right.$

$\left(-A+\frac{2 \sin \left[\frac{2 \pi}{q}\right]}{q}-\sin \left[\frac{2 \pi}{q}\right] A_{1}\right)+$

$\left(A+\frac{2 \operatorname{Sin}\left[\frac{2 \pi}{q}\right]}{q}-\operatorname{Sin}\left[\frac{2 \pi}{q}\right] A_{0}\right)$

$\left.\left.\left.\left(-\mathrm{A}+\frac{2 \operatorname{Sin}\left[\frac{2 \pi}{\mathrm{q}}\right]}{\mathrm{q}}-\operatorname{Sin}\left[\frac{2 \pi}{\mathrm{q}}\right] \mathrm{A}_{1}\right)\right)\right)\right)-$

$\frac{1}{8 \pi q^{4} \sqrt{1-R^{2}} a^{6}} A$

$\mathrm{E}^{\wedge}$

$\left(-\frac{\mathrm{A}^{2}}{2 \sigma^{2}}+\frac{1}{2\left(1-\mathrm{R}^{2}\right) \sigma^{2}}\right.$

$\left(\left(\frac{2 \operatorname{Cos}\left[\frac{2 \pi}{q}\right]}{q}-\operatorname{Cos}\left[\frac{2 \pi}{q}\right] A_{0}\right)^{2}+\right.$

$\left(A+\frac{2 \operatorname{Sin}\left[\frac{2 \pi}{q}\right]}{q}-\operatorname{Sin}\left[\frac{2 \pi}{q}\right] A_{0}\right)^{2}+$

$\left(-\frac{2 \cos \left[\frac{2 \pi}{q}\right]}{q}-\cos \left[\frac{2 \pi}{q}\right] A_{1}\right)^{2}+$

$\left(A+\frac{2 \operatorname{Sin}\left[\frac{2 \pi}{q}\right]}{q}-\operatorname{Sin}\left[\frac{2 \pi}{q}\right] A_{1}\right)^{2}-$

$2 \mathrm{R}$

$\left(\left(\frac{2 \operatorname{Cos}\left[\frac{2 \pi}{q}\right]}{q}-\cos \left[\frac{2 \pi}{q}\right] A_{0}\right)\right.$

$\left(\mathrm{A}+\frac{2 \sin \left[\frac{2 \pi}{q}\right]}{\mathrm{q}}-\sin \left[\frac{2 \pi}{\mathrm{q}}\right] \mathrm{A}_{1}\right)+$

$\left(A+\frac{2 \sin \left[\frac{2 \pi}{q}\right]}{q}-\sin \left[\frac{2 \pi}{q}\right] A_{0}\right)$

$\left.\left.\left.\left(A+\frac{2 \operatorname{Sin}\left[\frac{2 \pi}{q}\right]}{q}-\operatorname{Sin}\left[\frac{2 \pi}{q}\right] A_{1}\right)\right)\right)\right)$

Figure 7. The closed-form expression of symbol error probability

Testing convergence is done by making a different closed-form expression of symbol error probability of the $(q+1)^{\text {th }}$ and the $q^{\text {th }}$ member defined in Figure 8. The error function can be expressed in closed-form:

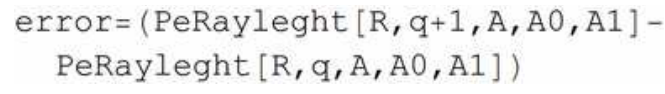

Figure 8. The error function of SEP

By testing of error function the number of iterations is obtained for need that error would be minimal. In fact, the minimum error represents the smallest change of error function in relation to the number of iterations. Figure 9 shows the dependence of the error functions in term of the number of iterations. In fact, it can be noted that correlation coefficient has the most impact on the calculation. In case when the correlation coefficient is small i.e. there is no correlation, it can be very quickly obtained 
an amount SEP regardless of the impact of interference. But, in case when the correlation coefficient is different from zero, especially in the area of high correlation (for example $R=0.95$ ) could be observed that the interference can affect the speed of calculation, on the way that the speed of calculation is greater when interference is present.

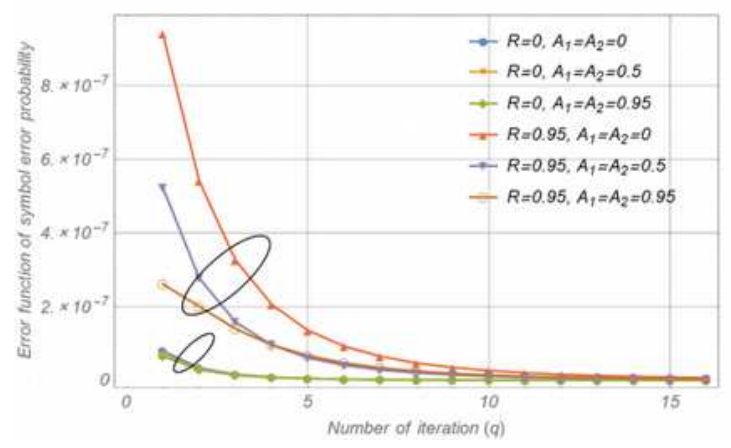

Figure 9. The error function of symbol error probability in term of number of iteration

Testing convergence examine in a simple manner provided by Wolfram language:

$$
\text { Limit [error, } q \rightarrow+\infty \text { ] }
$$

0

Figure 10. Testing convergence

In addition, we specify the acceptable error as $1 / 2^{b}$, where $b$ is the number of accurate bits. Solving the equation when error function is equal to required number of bits, we can determine $b$ in terms of the iteration index $q$.

$$
\begin{aligned}
& \text { sol } 4=\text { Solve }\left[\text { error }=\frac{1}{2^{b}}, b\right] / / \text { Flatten } \\
& \text { b /. sol } 4 / \text { change }
\end{aligned}
$$

Figure 11. The closed-form solution of number of bits in the term of iterations

Analytic form Figure 11 represents the closedform solution as relation between number of bits $b$ and number of iteration $q$, and it is shown on Figure 13. According to this, the number of accurate bits in term of number of iterations is shown on Figure 12. The simulation was performed assuming that interferences have account with $25 \%$ of amplitudes in regard to the useful signal. For optimal value of the number of iterations $(q=10)$ the range of the number of bits is from 23 bits for very strong correlated signal (taking $R=0.95$ ) to 27 bits for uncorrelated signal.

Figure 13 shows symbolic error probability in $\mathrm{dB}$, where $S N R=A /\left(2 \sigma^{2}\right)$, with optimized parameters such as the number of iterations.

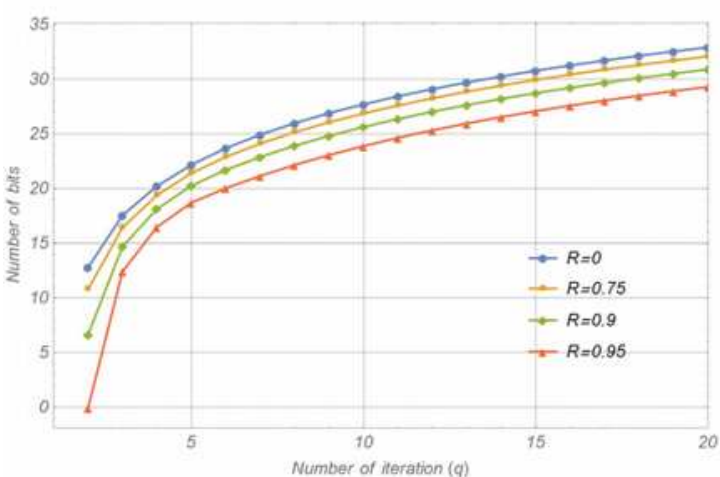

Figure 12. The functional dependence the number of bits in the term of iterations

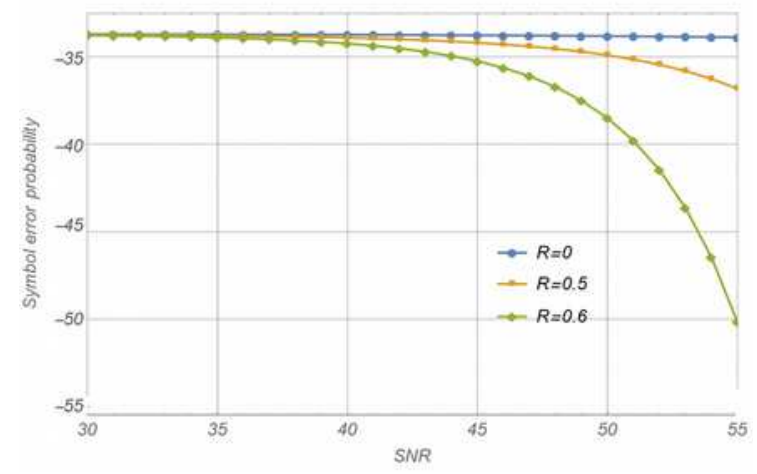

Figure 13. Symbol error probability (dB) with $q=10$ iterations, $b=23$ bits, variance $\sigma=3$, and $A_{1}=A_{2}=0.25$

The interferences in both branches of noncoherent FSK/ASK system has dominant influence in case of low correlation, but in opposite case when the correlation is high the impact to SEP becomes very evident. Moreover, it is significant to note that low change of correlation is having more significant influence on SEP.

The full expression that is shown on Figure 14 covers forth pages in format of this paper, so, we show a few terms as illustration the closedform solution in symbolic form that can be used for further analysis, manipulations, optimization or design.

\section{Conclusion}

This paper introduces development of a new method used for simulation of the processes and systems where there is no opportunity to represent mathematical formulas in the final form. Obtained solutions are applied to derive the symbol error probability needed for an analysis of performances of telecommunication systems. The presented iteration-based simulation method provides the opportunity for 


$$
\begin{aligned}
& \frac{1}{\log [2]} \log \left[\left(-\frac{32 \pi q^{4} \sigma^{6}}{\sqrt{1-R^{2}}}-\frac{128 \pi q^{5} \sigma^{6}}{\sqrt{1-R^{2}}}-\frac{192 \pi q^{6} \sigma^{6}}{\sqrt{1-R^{2}}}-\frac{128 \pi q^{7} \sigma^{6}}{\sqrt{1-R^{2}}}-\frac{32 \pi q^{8} \sigma^{6}}{\sqrt{1-R^{2}}}+\frac{32 \pi q^{4} R^{2} \sigma^{6}}{\sqrt{1-R^{2}}}+\right.\right. \\
& \left.\frac{128 \pi q^{5} R^{2} \sigma^{6}}{\sqrt{1-R^{2}}}+\frac{192 \pi q^{6} R^{2} \sigma^{6}}{\sqrt{1-R^{2}}}+\frac{128 \pi q^{7} R^{2} \sigma^{6}}{\sqrt{1-R^{2}}}+\frac{32 \pi q^{8} R^{2} \sigma^{6}}{\sqrt{1-R^{2}}}\right) / \\
& (A) \\
& E^{\wedge} \\
& \left(-\frac{A^{2}}{2 \sigma^{2}}+\frac{1}{2\left(1-R^{2}\right) \sigma^{2}}\left(\left(-\frac{\operatorname{Cos}\left[\frac{\pi}{q}\right]}{q}-\operatorname{Cos}\left[\frac{\pi}{q}\right] A_{0}\right)^{2}+\left(-A+\frac{\operatorname{Sin}\left[\frac{\pi}{q}\right]}{q}-\operatorname{Sin}\left[\frac{\pi}{q}\right]\right.\right.\right. \\
& \left.A_{0}\right)^{2}+\left(\frac{\operatorname{Cos}\left[\frac{\pi}{q}\right]}{q}-\operatorname{Cos}\left[\frac{\pi}{q}\right] A_{1}\right)^{2}+\left(-A+\frac{\operatorname{Sin}\left[\frac{\pi}{q}\right]}{q}-\operatorname{Sin}\left[\frac{\pi}{q}\right] A_{1}\right)^{2}-2 R \quad\left(\left(-\frac{\operatorname{Cos}\left[\frac{\pi}{q}\right]}{q}-\operatorname{Cos}\left[\frac{\pi}{q}\right]\right.\right. \\
& \left.A_{0}\right) \quad\left(-A+\frac{\operatorname{Sin}\left[\frac{\pi}{q}\right]}{q}-\operatorname{Sin}\left[\frac{\pi}{q}\right] A_{1}\right)+\left(-A+\frac{\operatorname{Sin}\left[\frac{\pi}{q}\right]}{q}-\operatorname{Sin}\left[\frac{\pi}{q}\right] A_{0}\right) \quad\left(-A+\frac{\operatorname{Sin}\left[\frac{\pi}{q}\right]}{q}-\operatorname{Sin}\left[\frac{\pi}{q}\right]\right. \\
& \left.\left.\left.\left.A_{1}\right)\right)\right)\right)+E^{\wedge}\left(-\frac{A^{2}}{2 \sigma^{2}}+\frac{1}{2\left(1-R^{2}\right) \sigma^{2}}\left(\left(-\frac{\operatorname{Cos}\left[\frac{\pi}{q}\right]}{q}-\operatorname{Cos}\left[\frac{\pi}{q}\right] A_{0}\right)^{2}+\left(-A+\frac{\operatorname{Sin}\left[\frac{\pi}{q}\right]}{q}-\operatorname{Sin}\left[\frac{\pi}{q}\right]\right.\right.\right. \\
& \left.A_{0}\right)^{2}+\left(\frac{\operatorname{Cos}\left[\frac{\pi}{q}\right]}{q}-\operatorname{Cos}\left[\frac{\pi}{q}\right] A_{1}\right)^{2}+\left(A+\frac{\operatorname{Sin}\left[\frac{\pi}{q}\right]}{q}-\operatorname{Sin}\left[\frac{\pi}{q}\right] A_{1}\right)^{2}-2 \operatorname{R}\left(\left(-\frac{\operatorname{Cos}\left[\frac{\pi}{q}\right]}{q}-\operatorname{Cos}\left[\frac{\pi}{q}\right] A_{0}\right)\right. \\
& \operatorname{Sin}\left[\frac{\pi}{q}\right] \\
& \left.\left.\left.\left(A+\operatorname{Sin}\left[\frac{\pi}{q}\right] A_{1}\right)+\left(-A+\frac{\operatorname{Sin}\left[\frac{\pi}{q}\right]}{q}-\operatorname{Sin}\left[\frac{\pi}{q}\right] A_{0}\right)\left(A+\frac{\operatorname{Sin}\left[\frac{\pi}{q}\right]}{q}-\operatorname{Sin}\left[\frac{\pi}{q}\right] A_{1}\right)\right)\right)\right)
\end{aligned}
$$

$4 \mathrm{E}^{\wedge}$

$$
\begin{aligned}
& \left(-\frac{A^{2}}{2 \sigma^{2}}+\frac{1}{2\left(1-R^{2}\right) \sigma^{2}}\left(\left(\frac{2 \operatorname{Cos}\left[\frac{2 \pi}{1+q}\right]}{1+q}-\operatorname{Cos}\left[\frac{2 \pi}{1+q}\right] A_{0}\right)^{2}+\left(A+\frac{2 \operatorname{Sin}\left[\frac{2 \pi}{1+q}\right]}{1+q}-\operatorname{Sin}\left[\frac{2 \pi}{1+q}\right]\right.\right.\right. \\
& \left.A_{0}\right)^{2}+\left(-\frac{2 \operatorname{Cos}\left[\frac{2 \pi}{1+q}\right]}{1+q}-\operatorname{Cos}\left[\frac{2 \pi}{1+q}\right] A_{1}\right)^{2}+\left(A+\frac{2 \operatorname{Sin}\left[\frac{2 \pi}{1+q}\right]}{1+q}-\operatorname{Sin}\left[\frac{2 \pi}{1+q}\right]\right. \\
& \left.A_{1}\right)^{2}-2 R\left(( \frac { 2 \operatorname { C o s } [ \frac { 2 \pi } { 1 + q } ] } { 1 + q } - \operatorname { C o s } [ \frac { 2 \pi } { 1 + q } ] A _ { 0 } ) \quad \left(A+\frac{2 \operatorname{Sin}\left[\frac{2 \pi}{1+q}\right]}{1+q}-\operatorname{Sin}\left[\frac{2 \pi}{1+q}\right]\right.\right. \\
& \left.\left.\left.\left.\left.\left.\left.A_{1}\right)+\left(A+\frac{2 \operatorname{Sin}\left[\frac{2 \pi}{1+q}\right]}{1+q}-\operatorname{Sin}\left[\frac{2 \pi}{1+q}\right] A_{0}\right) \quad\left(A+\frac{2 \operatorname{Sin}\left[\frac{2 \pi}{1+q}\right]}{1+q}-\operatorname{Sin}\left[\frac{2 \pi}{1+q}\right] A_{1}\right)\right)\right)\right) q^{4}\right)\right)\right]
\end{aligned}
$$

Figure 14. Closed-form solution of bits in terms of number of iteration

simplifications of complex calculations, manipulations of expressions, and deriving of accuracy in symbolic form. Programming knowledge is used immediately with Wolfram language as computer algebra system. The presented method has been done on the examples of digital telecommunication noncoherent FSK/ASK system.

\section{REFERENCES}

1. BORHANI, A., PATZOLD, M., Modelling of Vehicle-to-Vehicle Channels in the Presence of Moving Scatterers, Vehicular Technology Conference, 3-6 Sept. 2012, Quebec City, Canada, pp. 1-5.
2. BORWEIN, J. M., R. E. CRANDALL, Closed Forms: What They Are and Why We Care, Notices of the American Mathematical Society, vol. 60 (1), 2013, pp. 50-65.

3. HOGStad, B. O., C. A. GUTIERrEZ, M. PATZOLD, P. M. CRESPO, Classes of Sum-of-Cisoids Processes and Their Statistics for the Modelling and Simulation of Mobile Fading Channels, EURASIP Journal on Wireless Communications and Networking, vol. 2013(125), 2013, pp. 1-15.

4. HOGSTAD, B. O., M. PATZOLD, N. YOUSSEF, V. KONTOROVITCH, Exact 
Closed-Form Expressions for the Distribution, Level-Crossing Rate, and Average Duration of Fades of the Capacity of MIMO Channels, IEEE Transactions on Vehicular Technology, vol. 58 (2), 2008, pp. 1011-1016.

5. LI, W., NI, Q., Joint channel parameter estimation using evolutionary algorithm, IEEE ICC, 2010, Cape Town, South Africa, May 2010, pp. 1-6.

6. LUTOVAC, M., MLADENOVIC, V., LUTOVAC, M., Development of Aeronautical Communication System for Air Traffic Control Using OFDM and Computer Algebra Systems, Studies in Informatics and Control, vol. 22 (2), 2013, pp. 205-212.

7. MARTINEZ, W., A. MARTINEZ, Computational Statistics Handbook with MATLAB, Chapman \& Hall/CRC, 2002.

8. MLADENOVIC, V., M. LUTOVAC, M. LUTOVAC, Automated Proving Properties of Expectation-Maximization Algorithm Using Symbolic Tools, Telfor Journal, vol. 4 (1), 2012, pp. 54-59.

9. MLADENOVIC, V., M. LUTOVAC, D. PORRAT, Symbolic Analysis as Universal Tool for Deriving Properties of Nonlinear Algorithms-Case study of EM Algorithm, Acta Polytechnica Hungarica, vol. 11(2), 2014, pp. 117-136.
10. MLADENOVIC, V., D. MILOŠEVIC, A Novel-Iterative Simulation Method for Performance Analysis of Non-Coherent FSK/ASK Systems Over Rice/Rayleigh Channels using the Wolfram Language, Serbian Journal of Electrical Engineering, vol. 13 (2), 2016, pp. 157-174.

11. MLADENOVIC, V., M. LUTOVAC, Noncoherent FSK/ASK Signal Detection with Two Symbols Correlated Noise, $2^{\text {nd }}$ IcETRAN 2015, Silver Lake, Serbia, EKI 1.7, June 8-11, 2015, pp. 1-4.

12. MLADENOVIC, V., M. LUTOVAC, Simulation of Symbol-Error Ratio and Energy Efficiency using Computer Algebra Tools: A Case Study of OFDM Receiver, SINTESA Conferences, Belgrade, Serbia, April 2015, pp. 224-231.

13. PATZOLD, M., Mobile Radio Channels, $2^{\text {nd }}$ ed. Chichester, John Wiley \& Sons, 2011.

14. PAVLOVIC, V., V. MLADENOVIC, M. LUTOVAC, Telecommunications: Applications, Modern Technologies and Economic Impact, Book chapter, Nova Science Publishers, New York, USA, 2014.

15. WOLFRAM, S., An Elementary Introduction to the Wolfram Language, Wolfram Media, Inc, Campaign, USA, 2015. 